\title{
Assessment of Unmet Supportive Care Needs in Haematological Cancer Survivors
}

\author{
Theocharis I Konstantinidis ${ }^{1 *}$, Maria Spinthouri², Anastasia Ramoutsaki ${ }^{3}$, \\ Ariadni Marnelou ${ }^{4}$, George Kritsotakis ${ }^{1}$, Ourania Govina ${ }^{5}$
}

\begin{abstract}
Background: Health needs assessment is crucial for the provision of individualized nursing care. However, many patients report a significant number of unmet needs. The aim of the present study was the assessment of self-reported unmet supportive care needs among haematological cancer survivors in Greece. Methods: 102 patients (mean age 66.2 years old) diagnosed with haematological cancer were included in a cross-sectional study, conducted in two major Greek public hospitals, between October and December 2016. Patients' needs were assessed using the 'Needs Evaluation Questionnaire' (NEQ). Data analysis was conducted using the Statistical Package for Social Sciences software for Windows. Alfa-level ( $p$-value) selected was 5\%, bootstrap techniques were used for $95 \%$ CI estimation, $\chi^{2}$ was used for differentiation control and Kuder-Richardson coefficient for reliability score assessment $(\rho=0.922)$. Results: Patients reported higher needs levels "to receive less commiseration from other people" (48\%), "more information about my future condition" (44.1\%) and "to feel more useful within my family" (42.2\%). In contrast, patients reported lower levels to the needs "to speak with a spiritual advisor" (11.8\%), "to have more help with eating, dressing and going to the bathroom" (13.7\%) and "better attention from nurses" (18.6\%). The mean score of satisfied patients $(\geq 8 / 10)$ was 8.9 (SD 1.7). Associations between socio-demographic, hospitalization data and unmet needs groups were identified. The less satisfied patients $(<8 / 10)$ reported more informational needs about their diagnosis and their future condition ( $\mathrm{p}$-value $=0.002)$, about their exams and treatments $(\mathrm{p}$-value $=0.001)$, communicative ( $\mathrm{p}$-value $<0.001)$, assistance and treatment $(p$-value $<0.001)$ and hospital infrastructure ( $p$-value $<0.001)$. Conclusion: Various factors seem to be associated to the prevalent unmet care needs among haematological cancer patients. Establishing NEQ as a routine needs assessment tool could aid health professionals to early identify patients' needs in a busy clinical setting and implement more individualized and patient-centered quality care.
\end{abstract}

Keywords: Haematological cancer- needs evaluation questionnaire- unmet needs- supportive interventions

Asian Pac J Cancer Prev, 20 (5), 1487-1495

\section{Introduction}

Cancer patients report different levels of unmet needs through the disease trajectory (Bonacchi et al., 2018). A recent review found that the most frequent unmet care needs in patients with advanced cancer were informative (30-55\%), psychological (18-42\%), physical (17-48\%) and functional (17-37\%) (Moghaddam et al., 2016). Haematological cancer patients are a subgroup of total cancer population characterized by their heterogeneity in terms of diseases, therapy options, outcomes and life expectancy (Howell et al., 2011; Hall et al., 2015). They also report many unmet supportive needs related to the stage and type of their disease and therapy side effects (Boland et al., 2014; Boyes et al., 2015; Kim et al., 2017). Other predictors that may affect these needs include gender (Boyes et al., 2015), age (Boyes et al., 2015; Bron et al., 2017), advanced disease (Boland et al., 2014) and local health care services (Molassiotis et al., 2011). Furthermore, presumable needs such as satisfaction with hospital facilities, (bathrooms, food services, cleanliness, car parking accessibility), sexual functioning and the perceived support provided by doctors and nurses are not well documented through a typical needs' assessment (Molassiotis et al., 2011; Olsson et al., 2016). High number of unmet needs is associated with patients' distress and depression, burden their everyday functionality and decrease their quality of life (Molassiotis et al., 2011; Oberoi et al., 2017).

Early, continuous, systematic and accurate assessment of patients' and their families' needs and satisfaction is a core point to implement holistic care plans (Wen and 
Gustafson, 2004, Kritsotakis et al., 2010). It helps address patients' personal needs (Boyes et al., 2015) and manage the increased medical costs (Elkin and Bach, 2010). It also determines the needs that patients consider as important and immediate, highlights signs of inadequate assessment by health professionals and contributes to treatment adherence and satisfaction with care (Tamburini et al., 2000; Bonacchi et al., 2015; Hall et al., 2015).

Various tools for assessing supportive needs have been used in different groups of cancer patients (Hall et al., 2015; Kim et al., 2017; Tian et al., 2019). Research usually focus on needs of patients with solid tumors or from mixed cancer populations, while limited information is available on the needs of patients with specific haematological cancers (Hall et al., 2013; Swash et al., 2014; Konstantinidis et al., 2017). Questionnaires assessing needs concerning haematological cancer patients have been used for newly diagnosed patients, during treatment (Oberoi et al., 2017), advanced or end-stage patients (Boland et al., 2014), long-term survivors (Lobb et al., 2009; Kim et al., 2017) or to investigate needs in specific areas (Hall et al., 2015; Olsson et al., 2016).

In clinical environments with a high workload, time constraints, low numbers of health professionals and limited community oncology resources, such as in Greece, doctors and nurses not always ask about and patients do not easily report their needs and concerns beyond the physical and functional domain (Efstathiou et al., 2011). Thus, the use of short, easy-to-interpret, self-administered questionnaires, could make it easier to identify patients with unmet care needs that cause the greatest discomfort. In an effort to fill this gap, the aim of the present study was the assessment of unmet needs in a group of haematological cancer patients.

\section{Materials and Methods}

\section{Data collection process and participants}

All patients cared for as outpatients or as inpatients in the two biggest public general hospitals of Crete, Greece (one of them being the University hospital of the prefecture of Crete), were invited by the researchers face-to-face to participate in this cross-sectional study. These hospitals treat the vast majority of the haematological cancer patients in Southern Greece. From a total of 141 patients that were hospitalized during October and December 2016, a sample of 102 adult haematological cancer patients with leukemia, lymphoma Hodgkin's and non-Hodgkin's and multiple myeloma agreed to participate in the study (participation rate $72,3 \%$ ). Inclusion criteria were: age $>18$ years old, absence of psychiatric disorders, ability to write and speak in Greek and to complete forms.

\section{'Needs Evaluation Questionnaire'}

Patients completed the Greek version of the 'Needs Evaluation Questionnaire' (NEQ) (Konstantinidis et al., 2017). The initial version of NEQ was developed at the Istituto Nazionale Tumori in Italy and was mainly used to assess health care needs for support in hospitalized cancer patients (Tamburini et al., 2000; Tamburini et al., 2003).

NEQ is composed of 23 dichotomous items (yes/ no) that define eight different types of common needs: informational concerning diagnosis and prognosis (items 1 and 2) informational concerning examinations and treatment (items 3 and 4), communicative (items 5-8), relational needs (items 20-22), assistance and treatment (items 9-13), structure related needs (item 14), financial aspects (items 15 and 16), and needs for support (items 17-19, 23). A positive answer (yes) to at least one question in each needs category, was coded as a positive response to the respective category.

\section{Reliability analysis}

'Needs Evaluation Questionnaire' has already been translated and validated in Greek cancer patients showing adequate psychometric properties (Konstantinidis et al., 2017). A reliability analysis using the Kuder-Richardson statistic was performed in this sample before analyzing the data, also showing adequate psychometric properties $(\rho=0.922)$.

\section{Additional clinical and sociodemographic questions}

Personal and clinical data were also collected from medical records and the patients themselves (gender, age in years, marital status, education, place of residence, hospital visits during last two months, years from diagnosis etc.). Patient satisfaction was evaluated using a 10-point general satisfaction Likert-type scale. Mean time taken to complete the questionnaire was about $10 \mathrm{~min}$.

\section{Ethical considerations}

The study was approved by the Hospitals Ethics Committees' and all patients signed an informed consent letter prior to the participation in the study.

\section{Statistical Analysis}

Data were analyzed using the Statistical Package for Social Sciences (SPSS) software for Windows, version 23.0. The frequency distributions of basic descriptive characteristics were estimated, while the 23 needs were classified in order to determine the highest and lowest frequencies. 95\% confidence intervals of the relative frequency of the needs groups (at least one need from each group) were estimated using bootstrap techniques. Mann-Whitney and Kruskal-Wallis tests were used for the comparison of the non-parametric scores between needs and patients characteristics, because the scores were not normally distributed (Blom's method Q-Q plot).

Chi-square tests were used for the comparison of group of needs frequency between patients' characteristics (significant results are set with $\mathrm{p}<0.05$ ). In the sample of 102 patients there were 2 missing values in items 19 and 21. Missing values were replaced by the "mode" for each question.

\section{Results}

One hundred two patients (58.8\% males), of mean age 66.2 (SD 14.2) years, from two hospitals, completed all questionnaires. $69.6 \%$ were married, $58.8 \%$ had primary or no formal education, $40.2 \%$ were living in the city that the hospitals were established and $70.3 \%$ were living 
Table 1. Demographic, Clinical Data and Frequency of Needs of the 102 Haematological Cancer Patients

\begin{tabular}{|c|c|c|}
\hline & $\mathrm{n}$ & $\%$ \\
\hline \multicolumn{3}{|l|}{ Gender } \\
\hline Males & 60 & 58.8 \\
\hline Females & 42 & 41.2 \\
\hline \multicolumn{3}{|l|}{ Hospital } \\
\hline General non-University & 39 & 38.2 \\
\hline University general & 63 & 61.8 \\
\hline \multicolumn{3}{|l|}{ Age, years } \\
\hline up to 65 & 41 & 40.2 \\
\hline$\geq 65$ & 61 & 59.8 \\
\hline mean age (s.d.) [min-max] & \multicolumn{2}{|c|}{$66.2(14.2)[25-89]$} \\
\hline \multicolumn{3}{|l|}{ Marital status } \\
\hline Married & 71 & 69.6 \\
\hline Single & 10 & 9.8 \\
\hline Divorced, Widowed & 21 & 20.6 \\
\hline \multicolumn{3}{|l|}{ Education } \\
\hline No education & 8 & 7.8 \\
\hline Primary school & 52 & 51 \\
\hline Secondary school & 34 & 33.4 \\
\hline Higher (college/universities) & 8 & 7.8 \\
\hline \multicolumn{3}{|l|}{ Place of residence } \\
\hline Near hospital & 41 & 40.2 \\
\hline Other cities/ areas & 61 & 59.8 \\
\hline \multicolumn{3}{|l|}{ Living with } \\
\hline Husband/wife & 45 & 44.6 \\
\hline Husband/wife and children & 26 & 25.7 \\
\hline Children & 6 & 5.9 \\
\hline Parents/ brothers & 3 & 3 \\
\hline Alone & 21 & 20.8 \\
\hline \multicolumn{3}{|l|}{ Type of cancer } \\
\hline Lymphoma Hodgkin \& non-Hodgkin & 33 & 32.4 \\
\hline Acute and chronic leukemia & 50 & 49 \\
\hline Multiple myeloma & 19 & 18.6 \\
\hline \multicolumn{3}{|l|}{ Site of care ${ }^{a}$} \\
\hline Oncology ward & 56 & 62.9 \\
\hline Oncology day clinic & 33 & 37.1 \\
\hline \multicolumn{3}{|l|}{ Hospital visits during last two months ${ }^{b}$} \\
\hline Once & 26 & 26.5 \\
\hline Two & 24 & 24.5 \\
\hline Three, four & 20 & 20.4 \\
\hline Five or more & 28 & 28.6 \\
\hline \multicolumn{3}{|l|}{ Hospital days ${ }^{c}$} \\
\hline$\leq 6$ & 34 & 52.3 \\
\hline $7+$ & 31 & 47.7 \\
\hline mean (s.d.) $[\min -\max ]$ & $13.8(2$ & $1-210]$ \\
\hline \multicolumn{3}{|l|}{ Years from diagnosis ${ }^{\mathrm{d}}$} \\
\hline$<2$ & 44 & 46.8 \\
\hline $2+$ & 50 & 53.2 \\
\hline mean (s.d.) $[\min -\max ]$ & $4.2(5$ & $1-24.0]$ \\
\hline
\end{tabular}

Table 1. Continued

\begin{tabular}{|c|c|c|}
\hline & $\mathrm{n}$ & $\%$ \\
\hline \multicolumn{3}{|c|}{ Degree of satisfaction from care ${ }^{\mathrm{e}}$} \\
\hline$<8$ & 16 & 16.2 \\
\hline $8+$ & 83 & 83.8 \\
\hline mean (s.d.) [min-max] & \multicolumn{2}{|c|}{$8.9(1.7)[1-10]$} \\
\hline
\end{tabular}

with husband/wife and their children. The diagnoses were Hodgkin and non-Hodgkin lymphoma $(n=33,32.4 \%)$, acute and chronic leukemia $(\mathrm{n}=50,49 \%)$ and multiple myeloma $(n=19,18.6 \%) .53 .2 \%$ of patients had been diagnosed at least two years ago. The mean score of satisfied patients $(\geq 8 / 10)$ from the care provided was 8.9 $( \pm 1.7)$ (Table 1).

Patients reported higher levels to the needs "to receive less commiseration from other people" (48\%), "more information about my future condition" $(44.1 \%)$ and "to feel more useful within my family" (42.2\%). In contrast, patients reported lower levels of needs "to speak with a spiritual advisor" (11.8\%), "to have more help with eating, dressing and going to the bathroom)" (13.7\%) and "better attention from nurses" (18.6\%) (Table 2). Grouping needs identified by the NEQ, have shown that $66.7 \%$ of the patients expressed primarily 'support' needs (items 17 $19,23)$, followed by 'financial' $(61.8 \%)$ and 'relational' (55.9\%) needs (Figure 1).

Associations between socio-demographic, hospitalization data and needs groups are described in Table 3. Patients in the Non-University General Hospital reported more communicative $(64.1 \%$ vs $34.9 \%$ p-value $=$ $0.004)$ and hospital infrastructure needs (66.7\% vs. $25.4 \%$ p-value $<0.001$ ) than patients in University Hospital. Patients living near the hospital region expressed more informational needs (concerning diagnosis and prognosis) than those living in distant areas (61\% vs $37.7 \%$ p-value $=0.021)$. Patients who had been visited the hospital over 2-4 times during the last two months expressed less communication needs ( $p$-value $=0.033$ ) and support needs $(p$-value $=0.032)$ than the rest of the patients. Also, the less satisfied patients $(<8 / 10)$ reported more informational needs about their diagnosis and their future condition ( $p$-value $=0.002)$, informational needs about their exams and treatments ( $\mathrm{p}$-value $=0.001)$, communicative $(\mathrm{p}$-value $<0.001)$, assistance and treatment (p-value $<0.001)$ and hospital infrastructure ( $p$-value $<0.001$ ) than satisfied. Finally, patients with acute and chronic leukemia expressed more needs than those with other diseases ( $p$-value $=0.037$ ). Statistically significant differences were not observed among other variables such as sex, marital status, education, department of care years of diagnosis, cohabitation, days of hospitalization and years of diagnosis (p-value $>0.05$ ).

\section{Discussion}

In the present study we explored the unmet care needs in a group of haematological patients, using the NEQ, a 


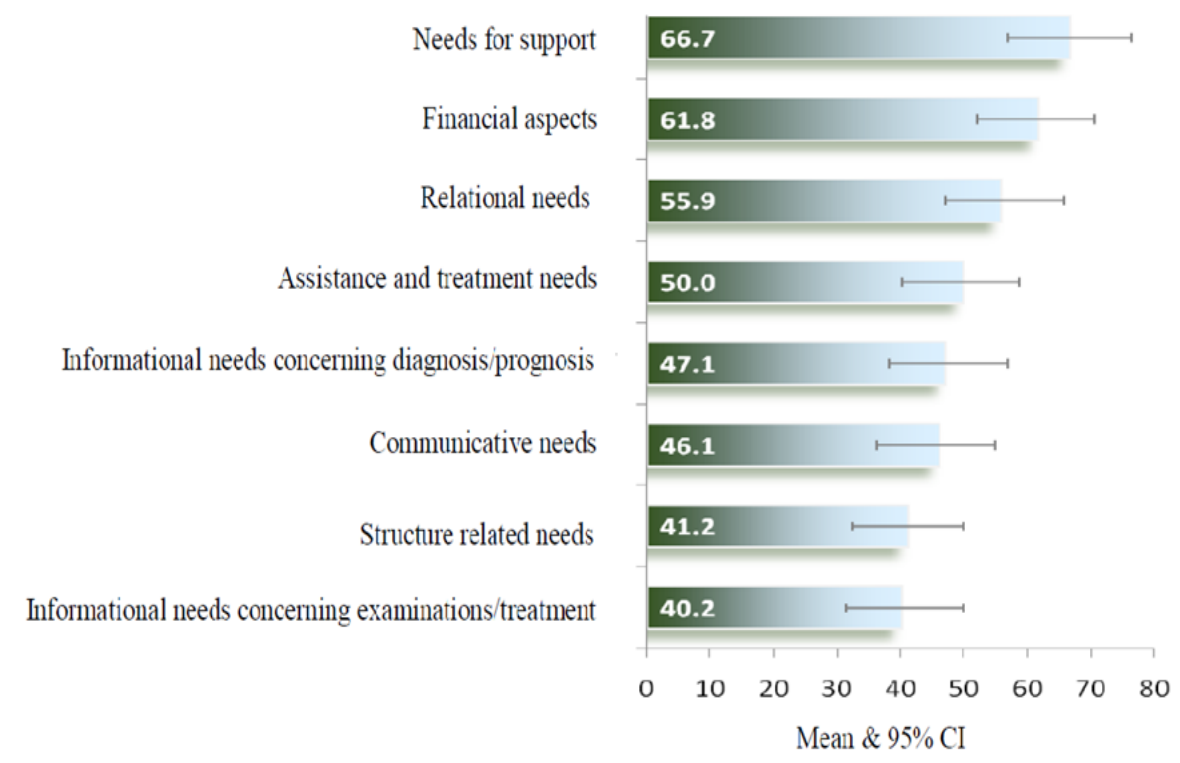

Figure 1. Classification of Unmet Needs of NEQ in Groups (at Least One Unmet Need in Each Group)

self-assessment needs tool. The mean number of reported unmet needs was equal to 7.3 and the dominant group of needs, according to the classification of Tamburini, was the needs for support (Tamburini et al., 2003). Similar findings with a high number of unmet needs have been found in a sample of leukemia and lymphoma survivors (Parry et al., 2012). In a recent study including only haematological cancer patients, more than half of the patients reported having at least one moderate to high unmet need (Boyes et al., 2015). Psychological, emotional, informational, physical and daily living needs, as well as needs related to the family and the hospital staff have been revealed in the majority of other similar studies (Lobb et al., 2009; Swash et al., 2014; Oberoi et

Table 2. Answers distribution of Needs Evaluation Questionnaire (NEQ) in 102 Cancer Patients

\begin{tabular}{|c|c|c|c|c|}
\hline Item & & Yes $(\%)$ & $\mathrm{n}$ & Higher/lower needs ${ }^{\alpha}$ \\
\hline 1 & I need more information about my diagnosis & 33.3 & 34 & 13 \\
\hline 2 & I need more information about my future condition & 44.1 & 45 & $2 \uparrow$ \\
\hline 3 & I need more information about the exams I am undergoing & 35.3 & 36 & 10 \\
\hline 4 & I need more explanations of treatments & 38.2 & 39 & 8 \\
\hline 5 & I need to be more involved in the therapeutic choices & 22.5 & 23 & $19 \downarrow$ \\
\hline 6 & I need clinicians and nurses to give me more comprehensible information & 33.3 & 34 & 12 \\
\hline 7 & I need clinicians to be more sincere with me & 30.4 & 31 & 15 \\
\hline 8 & I need to have a better dialogue with clinicians & 33.3 & 34 & 11 \\
\hline 9 & I need my symptoms (pain, nausea, insomnia, etc.) to be better controlled & 26.5 & 27 & 17 \\
\hline 10 & I need more help with eating, dressing, and going to the bathroom & 13.7 & 14 & $22 \downarrow$ \\
\hline 11 & I need better respect for my intimacy & 19.6 & 20 & $20 \downarrow$ \\
\hline 12 & I need better attention from nurses & 18.6 & 19 & $21 \downarrow$ \\
\hline 13 & I need to be more reassured by the clinicians & 24.5 & 25 & 18 \\
\hline 14 & I need better services from the hospital (bathrooms, meals, cleaning) & 41.2 & 42 & $6 \uparrow$ \\
\hline 15 & $\begin{array}{l}\text { I need to have more economic insurance information (tickets, invalidity, etc.) in } \\
\text { relation to my illness }\end{array}$ & 41.2 & 42 & $5 \uparrow$ \\
\hline 16 & I need economic help & 40.2 & 41 & 7 \\
\hline 17 & I need to speak with a psychologist & 26.5 & 27 & 16 \\
\hline 18 & I need to speak with a spiritual advisor & 11.8 & 12 & $23 \downarrow$ \\
\hline 19 & I need to speak with people who have the same experience & 41.2 & 42 & $4 \uparrow$ \\
\hline 20 & I need more reassured from my relatives & 31.4 & 32 & 14 \\
\hline 21 & I need to feel more useful within my family & 42.2 & 43 & $3 \uparrow$ \\
\hline 22 & I need to feel less abandoned & 35.3 & 36 & 9 \\
\hline 23 & I need to receive less commiseration from other people & 48 & 49 & $1 \uparrow$ \\
\hline
\end{tabular}

${ }^{\text {a }}$,With arrows are presented the five higher $(\uparrow)$ or lower $(\downarrow)$ needs according to percentages 
DOI:10.31557/APJCP.2019.20.5.1487

Needs Assessment in Haematological Malignancies

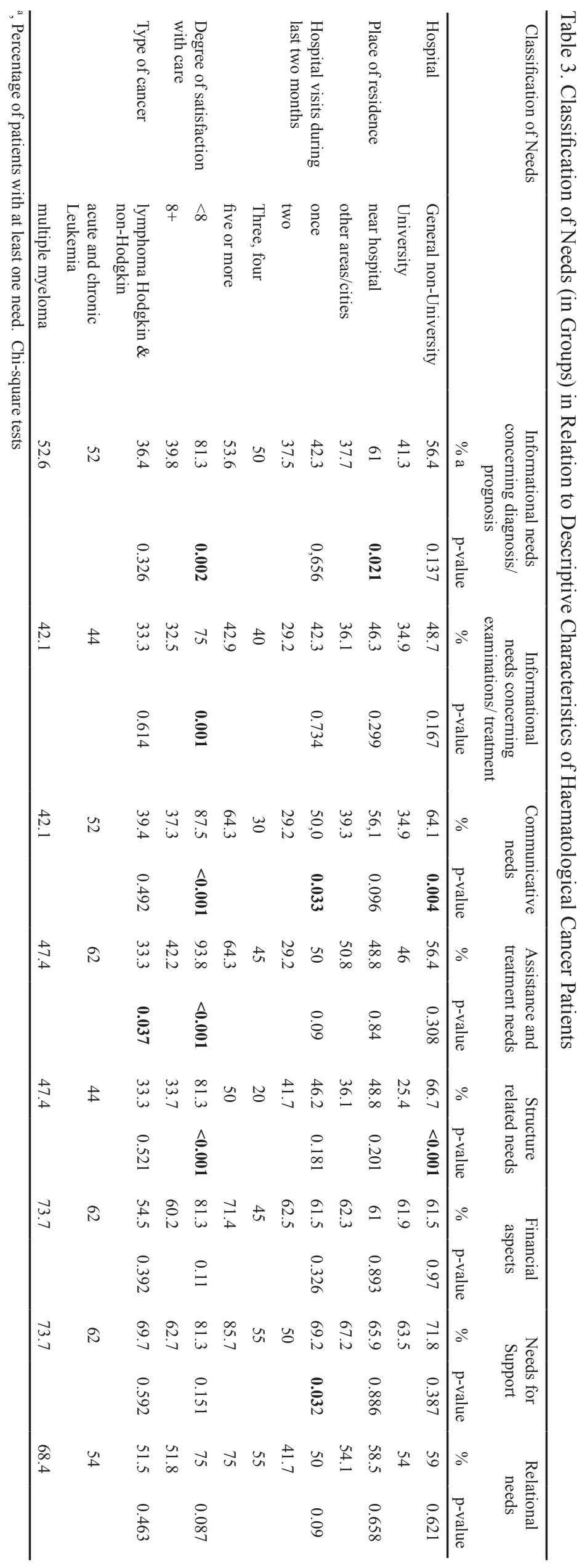


al., 2017). These needs vary in number and nature among different diagnostic groups of haematological cancer patients, due to quite often different symptoms, rate of progression, treatment and time of assessment after initial diagnosis (Manitta et al., 2011; Hall et al., 2014). They also usually differ from those in patients with solid tumors (Tamburini et al., 2000; Howell et al., 2011; Kim et al., 2017). Haematological cancer patients report relatively lower number of high unmet needs suggesting that the disease itself may cause less symptoms than in patients with other types of cancer or that the care provided is adequate and addresses to their needs (Hall et al., 2015).

The most frequently unmet need reported by patients in the present study was to receive less commiseration from other people, their families and close relatives. Findings from other studies indicate that in Greece and other Mediterranean countries, families and close relatives of cancer patients tend to feel more compassionate, suffer more, have closer and overprotective relationships with their patients and take care and support them at every point of their hospitalization (Sapountzi-Krepia et al., 2008). Thus, although there are no sufficient data, we can assume that our finding may indicate patients' concern to further protect their families from enormous stress resulting for their disease. Similarly, the above findings could also be associated to another finding of the present study, that patients reported lower levels of unmet needs during hospitalization in assisting in dressing, feeding and transportation to and from the hospital or they may be associated to better care by nurses.

The desire of patients to be more useful for their families was another highly reported unmet need in the present study. A probably quite relevant finding of another study was that patients with multiple myeloma reported that the way their families tried to help them was not always useful and sometimes they had to manage both their own and their families' reactions and feelings (Monterosso et al., 2018). The combination of the above findings could support the hypothesis that the interactive 'safety net' from families may increase the intensity of the highly reported relative needs and cause greater distress, anxiety and signs of depression to the families which in turn are being perceived by and affect negatively their patients (Molassiotis et al., 2011). Although further research is required to test all these hypotheses, it is important for health professionals not to underestimate caregivers concerns about their own problems and help them interact with their important ones, by providing appropriate information about patients' care, supportive care services and social support, on an ongoing basis (Lund et al., 2015).

In the present study, patients did not report a significant amount of informational needs for diagnosis, examinations and treatment which has been found common in other studies with patients with solid tumors (Tamburini et al., 2003; Konstantinidis et al., 2017). High levels of informational needs focusing on haemaetological patients in this area are not very often recorded (Swash et al., 2014; Yu et al., 2017). Usually studies have revealed high informational needs related to better informed about their future situation which is primarily associated with fear of recurrence (Kim et al., 2017). This fear is rated by the patients as one of the prevalent unsatisfied needs involving not only mixed haematological cancer populations but also patients with solid tumors (Swash et al., 2014; Ozga et al., 2015). Fear remains after the cancer treatment and may be accompanied by psychological distress and decreased quality of life (Simard et al., 2013). In a recent study of 826 Korean survivors with non-Hodgkin lymphoma, information about prevention of recurrence and metastasis were the two greatest needs (Kim et al., 2017). Cancer education, providing effective information and referring patients timely for psychological support may be helpful to improve their suffering.

In the present study, patients also expressed an increased need for support groups where patients could speak with people who had the same experience. Supportive teams play an important role in the overall care of patients and their families and may provide help to care-related decisions, reduce thoughts about death and dying and resolve other everyday life issues related to sexuality, community supportive structures, side effects, anxiety and depression and future adaption to the disease (Butow et al., 2013; Hoffman et al., 2013). Although discussions with others, especially of similar age and status, may be helpful to some patients, some others may find such discussions quite stressful, indicating that patient's willingness should be previously considered (Monterosso et al., 2018).

Psychological distress such as depression and anxiety are consistent findings in the literature and require supportive care services (Molassiotis et al., 2011; Oberoi et al., 2017). Furthermore, care plans for haematological patients may include effective interventions for the reported requests of patients who wish having access to an ongoing case manager (Lobb et al., 2009; Molassiotis et al., 2011), dealing with lack of energy/feeling tired (Boyes et al., 2015; Hall et al., 2015; Tzelepis et al., 2018), practical problems such as accessibility to hospital parking (Molassiotis et al., 2011), handling medical expenses, health insurance and employment problems (Parry et al., 2012) and physical and daily living needs (Oberoi et al., 2017). However, there is little evidence about the effectiveness of such interventions in reducing unmet needs mainly because of psychometric inadequacies of the interventional tools, small sample sizes, scoring problems, low response rates and ineffective interventions (Carey et al., 2012; Hall et al., 2013).

Patients in the current study did not report a high need for discussions with a spiritual advisor. This could be associated either to a low level of spiritual need per se or to a distorted image in Greek patients that spiritual needs concern only the terminally ill patients. It may also be related to the fact that spirituality, and religiousness in particular, is mainly supported by not well-trained priests or they are not often reported or investigated as a possible unmet need by health professionals. Further investigation is required in that field as a recent study highlighted the positive impact of spiritual wellbeing in patient symptoms, effective coping mechanisms and improved quality of life 
suggesting (Ripamonti et al., 2018).

There were no significant differences in patients' needs in this study comparing with factors such as gender, age and educational level. However, significantly greater unsatisfied needs have been reported in younger patients, females, unmarried and low monthly income in many other studies (Lobb et al., 2009; Parry et al., 2012; Boyes et al., 2015; Kim et al., 2017). Younger age and female sex are also predictors for higher unmet needs in advanced cancer patients with solid tumors (Konstantinidis et al., 2017).

Patient's satisfaction was not in itself an indicator of good care in the present study where patients appeared to be satisfied with the care provided by nursing staff despite their increased number of needs. This may be attributed to their hospitalization in well-established oncology departments or to their families' continuing support in every step of their care. Moreover, some patients may not consider the role of nurses in meeting their needs mainly because of the way the nursing is exerted that creates low expectations or claims, particularly in the reported main unmet needs. However, it has been well documented that the overall increased number of needs imply an inadequate understanding by health personnel of the self-reported, patient's priorities related needs and highlights the need to be approached by a multidisciplinary well-trained oncology team (Bahrami and Arbon, 2012; Oberoi et al., 2017).

The three major group of needs the patients reported in this study were for support, financial aspects and relational. Support needs, as identified by NEQ, are common between haematological patients, especially during and after treatment. Assessment with questionnaires and interviews may be helpful for patients completing the acute phase of treatment to find out needs that have not been well addressed yet (Lobb et al., 2009).

Similarly to other studies, financial aspects were an important concern for many patients in the present study mainly for rural habitants of low income who travel long distance to visit hospitals (Yu et al., 2017; Tzelepis et al., 2018). Further investigation of medical and other indirect and often underestimated costs could help redesigning the interventions and policies required to manage these unmet needs and reduce the overall disease burden (Elkin and Bach, 2010; Hall et al., 2014; Hall et al., 2015). As mentioned above, relatives play a significant role in patients care. Overwhelming families should be supported by health professionals because their reactions, feelings, actions and everyday functionality significantly affect patients' wellbeing and unmet needs in many different ways (Kim et al., 2008).

Increased visits/hospitalizations in the last two months were characterized by a high number of communication and support needs, indicating that these patients may require more intensive treatment or are in a more advanced stage resulting in increased number of such needs. Patients with leukemia reported significantly higher unmet supportive care needs in assistance and treatment group of needs. These patients have a substantial symptom burden, especially in the acute phase. However, different results among haematological patients have been found in the literature because needs are associated with the multiple arising symptoms due to the different stage of the diseases, the kind of needs assessment tool used and the intensity of treatments (Molassiotis et al., 2011; Dyson et al., 2012; Hall et al., 2013; Zimmermann et al., 2013).

\section{Limitations}

There are some limitations in the present study. Comparisons between the prevalence of needs among patients with different hematological malignancies could not be made in detail because of the small sample size of those subgroups. Furthermore, generalization should be made by caution since all patients came from the region of Crete, Greece, although these hospitals are the main oncology and hematology centers in Southern Greece. Finally, this is the first use of NEQ in Greek hematological cancer patients and, because of its design, we can assume that covers only basic patients' needs and acts more as a screening test for identifying patients who need further investigation. Future studies are needed to confirm and extend the current results and assess the suitability of the instrument among different cancer patients and research designs.

In conclusion, in the current study, a wide range of unmet supportive care needs were identified in a group of haematological cancer patients using a simple and easy to administer self-reported questionnaire. Patients endorsed significantly more needs around emotional and relationship issues focusing mainly in the way patients interact with their family and the informational gap about their future condition expressing their fear for recurrence. They also reported needs in the domains of supportive, financial and relational issues. Many factors such as the number of hospitalizations, the disease, the local health conditions and the place of residence can have a significant impact on certain categories of needs. Nevertheless, patients appear to be satisfied with the nursing care.

\section{Implications}

The routine use of NEQ in a busy clinical setting can help health care professionals to early and easily identify individualized patients' needs and expectations, to achieve optimal, individualized, patient-centered care, enhance their quality of life and reduce cost of care.

\section{Acknowledgements}

The study was not funded by any research body. Authors declare no conflict of interest. The study was approved by the Hospitals Ethics Committees' and all patients signed an informed consent letter prior to the participation in the study.

\section{References}

Bahrami M, Arbon P (2012). How do nurses assess quality of life of cancer patients in oncology wards and palliative settings? Eur J Oncol Nurs, 16, 212-9.

Boland EG, Boland JW, Ezaydi Y, et al (2014). Holistic needs assessment in advanced, intensively treated multiple 
myeloma patients. Support Care Cancer, 22, 2615.

Bonacchi A, Toccafondi A, Mambrini A, et al (2015). Complementary needs behind complementary therapies in cancer patients. Psychooncology, 24, 1124-0.

Bonacchi A, Di Miceli S, Lippi D, Muraca MG, Miccinesi G (2018). Unmet needs of Italian cancer patients in different stages of the disease and care process. Tumori, 104, 285-1.

Boyes AW, Clinton-McHarg T, Waller AE, et al (2015). Prevalence and correlates of the unmet supportive care needs of individuals diagnosed with a haematological malignancy. Acta Oncol, 54, 507-4.

Bron D, Aurer I, André MPE, et al (2017). Unmet needs in the scientific approach to older patients with lymphoma. Haematologica, 102, 972-5.

Butow P, Price MA, Shaw JM, et al (2015). Clinical pathway for the screening, assessment and management of anxiety and depression in adult cancer patients: Australian guidelines. Psychooncology, 24, 987-1.

Carey M, Lambert S, Smits R, et al (2012). The unfulfilled promise: a systematic review of interventions to reduce the unmet supportive care needs of cancer patients. Support Care Cancer, 20, 207-9.

Dyson GJ, Thompson K, Palmer S, Thomas DM, Schofield P (2012). The relationship between unmet needs and distress amongst young people with cancer. Support Care Cancer, 20, 75-5.

Efstathiou N, Coll AM, Ameen J, Daly W (2011). Do Greek healthcare users and healthcare providers share cancer care priorities? Analysing the results from two Delphi studies. Eur J Cancer Care, 20, 179-6.

Elkin EB, Bach PB (2010). Cancer's next frontier: addressing high and increasing costs. JAMA, 303, 1086-7.

Hall A, Lynagh M, Bryant J, Sanson-Fisher R (2013). Supportive care needs of hematological cancer survivors: a critical review of the literature. Crit Rev Oncol Hematol, 88, 102-6.

Hall A, D’Este C, Tzelepis F, Lynagh M, Sanson-Fisher R (2014). Factors associated with haematological cancer survivors experiencing a high level of unmet need across multiple items of supportive care: a cross-sectional survey study. Support Care Cancer, 22, 2899-9.

Hall AE, Sanson-Fisher RW, Lynagh MC, Tzelepis F, D’Este C (2015). What do haematological cancer survivors want help with? A cross-sectional investigation of unmet supportive care needs. BMC Res Notes, $\mathbf{8}, 221$.

Hoffman MA, Lent RW, Raque-Bogdan TL (2013). A social cognitive perspective on coping with cancer: Theory, research, and intervention. Couns Psychol, 41, 240-7.

Howell DA, Shellens R, Roman E, et al (2011). Haematological malignancy: are patients appropriately referred for specialist palliative and hospice care? A systematic review and meta-analysis of published data. Palliat Med, 25, 630-1.

Kim SH, Lee S, Kim SH, et al (2017). Unmet needs of non-Hodgkin lymphoma survivors in Korea: prevalence, correlates, and associations with health-related quality of life. Psychooncology, 26, 330-6.

Kim Y, Kashy DA, Wellisch DK, et al (2008). Quality of life of couples dealing with cancer: dyadic and individual adjustment among breast and prostate cancer survivors and their spousal caregivers. Ann Behav Med, 35, 230-8.

Konstantinidis T, Samonis G, Sarafis P, Philalithis A (2017). Assessment of needs of hospitalized cancer patients with advanced cancer. Glob J Health Sci, 9, 184-4.

Kritsotakis G, Koutis A, Kotsori A, Alexopoulos C, Philalithis A (2010). Measuring patient satisfaction in oncology units: interview based psychometric validation of the 'Comprehensive Assessment of Satisfaction with Care' in Greece. Eur J Cancer Care, 19, 45-2.
Lobb EA, Joske D, Butow P, et al (2009). When the safety net of treatment has been removed: patients' unmet needs at the completion of treatment for haematological malignancies. Patient Educ Couns, 77, 103-8.

Lund L, Ross L, Petersen MA, Groenvold M (2015). The interaction between informal cancer caregivers and health care professionals: a survey of caregivers' experiences of problems and unmet needs. Support Care Cancer, 23, 1719-3.

Manitta V, Zordan R, Cole-Sinclair M, Nandurkar H, Philip J (2011). The symptom burden of patients with hematological malignancy: a cross-sectional observational study. J Pain Symptom Manage, 42, 432-2.

Moghaddam N, Coxon H, Nabarro S, Hardy B, Cox K (2016). Unmet care needs in people living with advanced cancer: a systematic review. Support Care Cancer, 24, 3609-2.

Molassiotis A, Wilson B, Blair S, Howe T, Cavet J (2011). Unmet supportive care needs, psychological well-being and quality of life in patients living with multiple myeloma and their partners. Psychooncology, 20, 88-7.

Monterosso L, Taylor K, Platt V, et al (2018). Living with multiple myeloma: A focus group study of unmet needs and preferences for survivorship care. J Patient Exp, 5, 6-5.

Oberoi DV, White VM, Seymour JF, et al (2017). Distress and unmet needs during treatment and quality of life in early cancer survivorship: A longitudinal study of haematological cancer patients. Eur J Haematol, 99, 423-0.

Olsson C, Sandin-Bojö AK, Bjuresäter K, Larsson M (2016). Changes in sexuality, body image and health related quality of life in patients treated for hematologic malignancies: A longitudinal study. Sex Disabil, 34, 367-8.

Ozga M, Aghajanian C, Myers-Virtue S, et al (2015). A systematic review of ovarian cancer and fear of recurrence. Palliat Support Care, 13, 1771-0.

Parry C, Lomax JB, Morningstar EA, Fairclough DL (2012). Identification and correlates of unmet service needs in adult leukemia and lymphoma survivors after treatment. J Oncol Pract, 8, e135-1.

Ripamonti CI, Giuntoli F, Gonella S, Miccinesi G (2018). Spiritual care in cancer patients: a need or an option?. Curr Opin Oncol, 30, 212-8.

Sapountzi-Krepia D, Raftopoulos V, Psychogiou M, et al (2008). Dimensions of informal care in Greece: the family's contribution to the care of patients hospitalized in an oncology hospital. J Clin Nurs, 17, 1287-4.

Simard S, Thewes B, Humphris G, et al (2013). Fear of cancer recurrence in adult cancer survivors: a systematic review of quantitative studies. J Cancer Surviv, 7, 300-2.

Swash B, Hulbert-Williams N, Bramwell R (2014). Unmet psychosocial needs in haematological cancer: a systematic review. Support Care Cancer, 22, 1131-1.

Tamburini M, Gangeri L, Brunelli C, et al (2000). Assessment of hospitalised cancer patients' needs by the Needs Evaluation Questionnaire. Ann Oncol, 11, 31-7.

Tamburini M, Gangeri L, Brunelli C, et al (2003). Cancer patients' needs during hospitalization: a quantitative and qualitative study. BMC Cancer, $\mathbf{3}, 12$.

Tian L, Cao X, Feng X (2019). Evaluation of psychometric properties of needs assessment tools in cancer patients: A systematic literature review. PLoS One, 14, e0210242.

Tzelepis F, Paul CL, Sanson-Fisher RW, et al (2018). Unmet supportive care needs of haematological cancer survivors: rural versus urban residents. Ann Hematol, 97, 1283-2.

Wen KY, Gustafson DH (2004). Needs assessment for cancer patients and their families. Health Qual Life Outcomes, $2,11$.

Yu FF, Bai YN, He H, et al (2017). Identifying the unmet supportive care needs, with concomitant influencing factors, 
in adult acute leukemia patients in China. Eur J Oncol Nurs, 30, 67-4.

Zimmermann C, Yuen D, Mischitelle A, et al (2013). Symptom burden and supportive care in patients with acute leukemia. Leuk Res, 37, 731-6.

\section{c) (i) (8)}

This work is licensed under a Creative Commons AttributionNon Commercial 4.0 International License. 